\title{
دراسة تحليلية لأهم العوامل المؤئرة على واردات مصر من القمح
}

\author{
حمدي سيا عبده عبد العال ، أحمد محمد عبد العزيز الشافعي \\ قسم الاقتصاد الزراعي، كلية الزراعة، جامعة المنيا
}

Received: Aug. 25, 2021

Accepted: Sep. 20, 2021

الملخص

يعتبر القمح من أهم المحاصيل الزراعية وأهم سلع الواردات الغذائية في مصر ـ ولسنوات عديدة، اعتمدت مصر بشكل

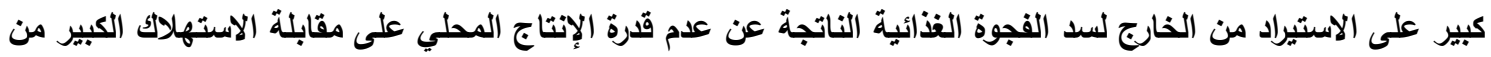

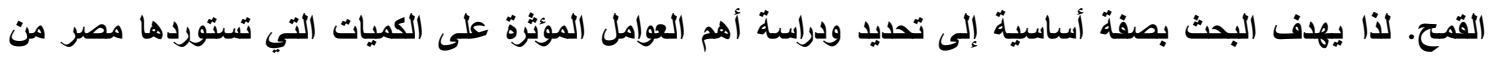

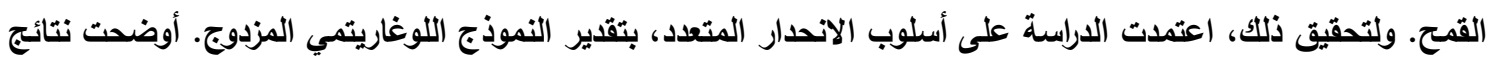
الاراسة أن واردات القمح تمثل نسبة كبيرة من الواردات الغذائية بلغت في المتوسط 23.19\% من إجمالي الواردات

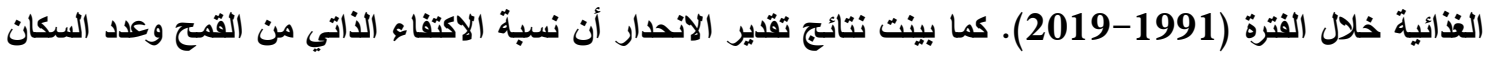

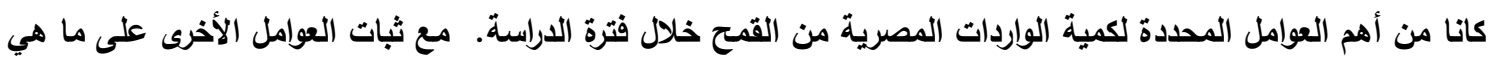

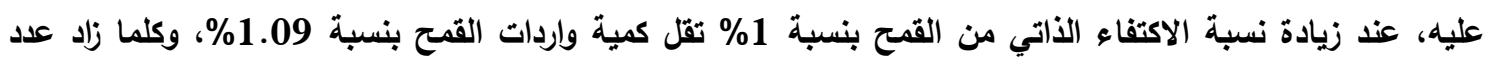
السكان بنسبة 1\% تزيد كمية واردات القمح بنسبة 0.85\%. لذا توصي الدراسة بضرورة ترشيد الاستهلاك من القمح والعمل علي زيادة الإنتاج المحلي من القمـح.

الكلمات المفتاحية: واردات القمح- نسبة الاكتفاء الذاتي- عدد السكان - الفجوة الفذائية

عام 2019، شكلت واردات مصر الغذائية حوالي 20٪ المقدمة

من إجمالي وارداتها (منظمة التجارة العالمية، 2019 2019). أما في المستقبل، فمن المتوقع أن يزداد اعتماد الدولة على الواردات الغذائية لتلبية حاجة السكان من الغذاء؛ إذا أخنا في الاعتبار النمو السكاني الكبير، ومحدودية

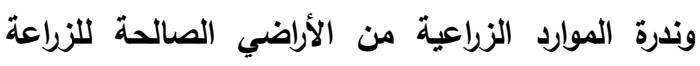

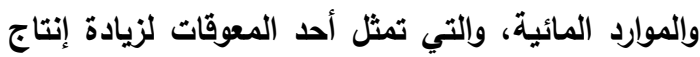
الغذاء مستقبلا. ويعتبر القمح أحد أهم المحاصيل الاستراتيجية في مصر الأي يعتمد عليه معظم السكان في نظامهم الغذائي اليومي، ويحتل المرتبة الأولي بين المحاصيل الزراعية من حيث المساحة المزروعة، حيث بلغت المساحة المزروعة بالقمح في 2018 حوالي 3.2 مليون فدان تمثل 20\% من إجمالي المساحة المحصولية، وحوالي 35 من إجمالي المساحة المنزرعة في الموسم الثتوي المئل
علي الرغم من أن مصر قايما، خاصة في عهر

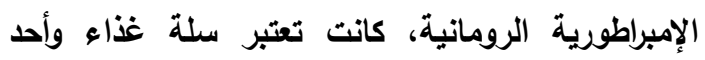

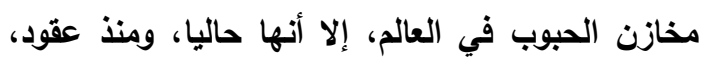

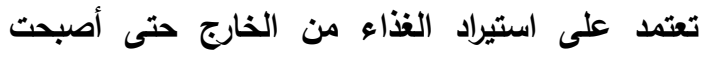

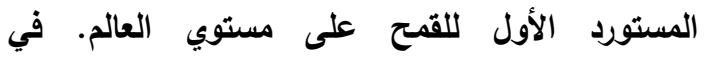
الستينيات، كانت مصر تنتج معظم احتياجاتها الغذائية الأساسية، باستثناء القمح الذي كانت تستورد منه حوالي

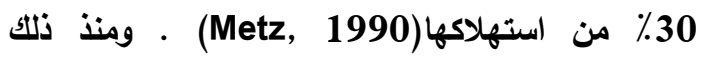

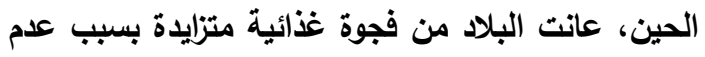

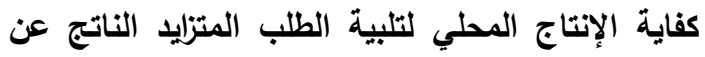
النمو السكاني الكبير والسريع. وفي الآونة الأخيرة، ارتفعت واردات مصر من المواد الغذائية لتثكل حصة الاونه الآيرة

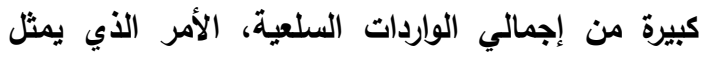

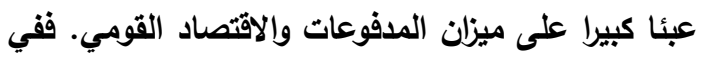


يهدف البحث بصفة أساسية إلى تحديد ودراسة أهم

العوامل المؤثرة على الكميات التي تستوردها مصر من القمح خلال فترة الدراسة (1991-2019). كما يهدف البحث إلى التعرف على الأهمية النسبية لقيمة الواردات الغذائية من قيمة الواردات الكلية، وكذلك الأهمية النسبية لقيمة واردات القمح من قيمة الواردات الغذائية.

$$
\text { الطريقة البحثية ومصادر البيانات }
$$

للتعرف على الأهمية النسبية لقيمة واردات الغذاء من قيمة الواردات الكلية، والأهمية النسبية لقيمة واردات

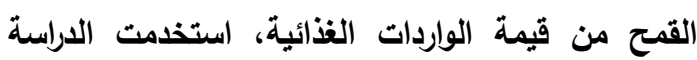
بعض الأساليب الوصفية مثل الجداول والرسوم البيانية والمتوسطات والنسب المئوية. وتم الحصول على بيانات قيمة الواردات الكلية لمصر (مليار دولار)، وقيمة واردات مصر من الغذاء (مليار دولار) من موقع منظمة التجارة

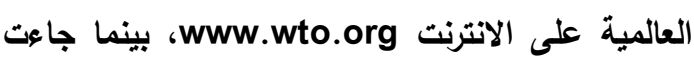
بيانات قيمة واردات مصر من القمح من موقع منظمة .www.fao.org/faostat/en/\#home الفاو ولدراسة وتحديد أهم العوامل المؤثرة على الكمية المستوردة من القمح، اعتمدت الدراسة على أسلوب الانحدار المتعدد، بتقدير النموذج اللوغاريتمي المزدوج، وذلك باستخدام برنامج EViews. $\ln \mathrm{Wm}_{i}=\beta_{0}+\beta_{1} \operatorname{lnPop}{ }_{i}+\beta_{2} \operatorname{lnS} r_{i}+\beta_{3} \ln P r_{i}$

$$
+\beta_{4} D
$$

حيث ln هي أساس اللوغاريتم الطبيعي، Wm

كمية واردات القمح بالمليون طن في السنةiة، هو عدد السكان (مليون نسمة) في السنةi

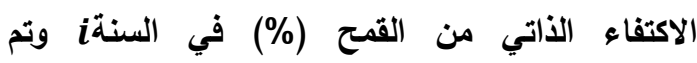
استخدامها لأنها تعكس الفجوة بين العرض المحلي من القمح (الإنتاج الكلي) والطلب علي القمح (الاستهلاك)، وتم حسابها بقسمة الإنتاج المحلي من القمح في سنة ما على الاستهلاك من القمح في نفس السنة، ${ }^{\text {ه }}$ النسبة السعرية بين السعر المحلي بالدولار للطن في السنة i والسعر العالمي بالدولار للطن في نفس السنة، D
في نفس العام. كما تستورد مصر منه كميات كبيرة سنويا، حيث تعتبر من الدول الرئيسية المستوردة للقمح على مستوي العالم، وجاءت في المرتبة الأولي من حيث الكميات المستوردة عالميا خلال العشر سنوات الأخيرة (2010-2019) بمتوسط واردات سنوية بلغت حوالي 10.5 مليون طن، تثكل حوالي 6.20\% من متوسط FAO, ) الكميات المستوردة عالميا خلال نفس القترة .$(2021$

وتوضح بيانات منظمة الفاو أن روسيا جاءت في مقدمة الدول المصدرة للقمح إلى مصر خلال الفترة (2015-2019)، حيث بلغ المتوسط السنوي لكمية واردات القمح المصرية القادمة منها حوالي 5.83 مليون طن، يمثل حوالي 54\% من متوسط كميات القمح التي تستوردها مصر سنويا من جميع دول العالم، والبالغ حوالي 10.7 مليون طن خلال نفس القترة. وجاءت كل من أوكرانيا ورومانيا في الترتيب الثاني والثالث بين أهم الاول المصدرة للقمح إلى مصر، حيث بلغ متوسط كمية واردات القمح من هذه الدول حوالي 1.84، 1.09 مليون طن، يمثل حوالي 17\%، 10\% من متوسط كميات القمح التي تستوردها مصر سنويا من جميع دول العالم على الترتيب خلال نفس الفترة (FAO, 2021). مشكية البحث منذ عدة سنوات، اعتمدت مصر بشكل كبير على الإنى الاستيراد من الخارج لسد الفجوة الغذائية الناتجة عن عدم قرة الإنتاج المحلي على مقابلة الاستهلاك الكبير من الغذاء خاصة من القمح. إن زيادة الاعتماد على الواردات الغذائية يزيد من تعرض البلاد لتقلب أسعار الغذاء العالمية، علاوة علي زيادة العجز في الميزان التجاري للاولة، كما يتطلب وجود اقتصاد قوي وتوافر احتياطيات كبيرة من النقد الأجنبي لدعم الإنفاق على الواردات الغذائية الرئيسية، مثل القمح. أهداف الدراسة 
فإن القرارات المتعلقة بتخصيص النقد الأجنبي لاستيراد

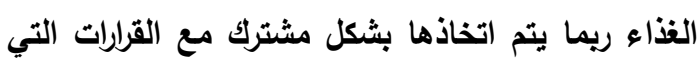

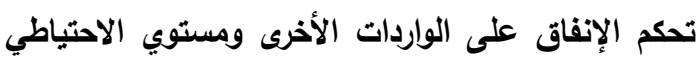

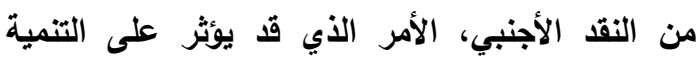
الاقتصادية مستقبلا.

2- الأهمية النسبية لقيمة واردات القمح من قيمة الواردات الغذائية

يعتبر القمح من أهم محاصيل الحبوب وأهم سلع

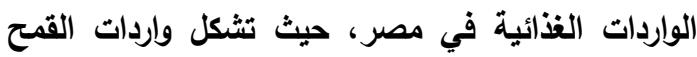

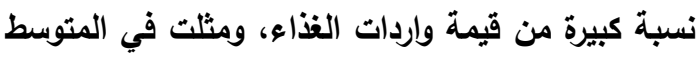
حوالي 23.19\% من متوسط إجمالي قيمة واردات

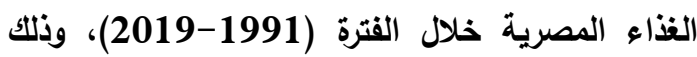
كما يتضح من جدول (1). وخلال فترة الدراسة، تراوحت قيمة واردات مصر من القمح ما بين 500 مليون دولار

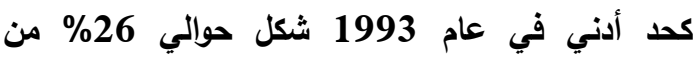

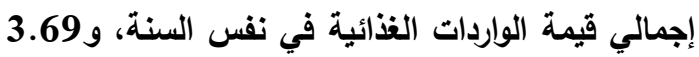
مليار دولار كد أعلي في عام 2012 شكل حوالي 23 من إجمالي قيمة الواردات الغذائية في نفس فئس السنة. كما بلغ متوسط قيمة واردات مصر من القمح

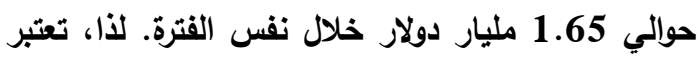

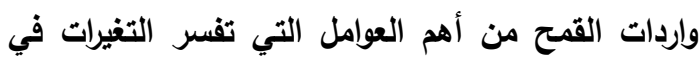
قيمة الواردات الغذائية، حيث يلاحظ من جدول وشكل (2) أن قيمة الواردات الغذائية وصلت لحدها الأدنى في عام 1993 عندما وصلت قيمة واردات القمح لأقل قيمة في نفس العام، كما وصلت قيمة الواردات الغذائية إلى أقصى قيمة لها في عام 2012 عندما بلغت قيمة واردات القمح حدها الأقصى في نفس العام.

$$
\text { 3- تطور كمية الواردات من القمح }
$$

بدراسة تطور كمية واردات مصر من القمح خلال

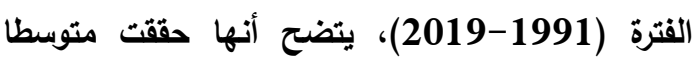

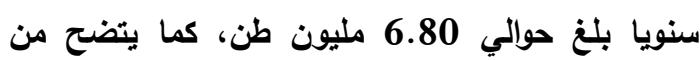
بيانات جدول (3). ويمتابعة بيانات ذات الجدول، يتبين أن كمية واردات القمح تراوحت بين 3.85 مليون طن
الإنتاج والاستهلاك من القمح، ويأخذ القيمة "صفر"

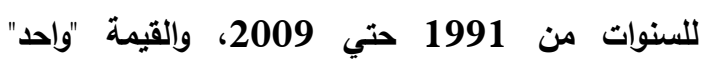
للسنوات من 2010 حتي 1919. وتم الحصول على البيانات اللازمة لتقدير النموذج من مصادر متعددة، حيث جاهت بيانات كمية واردات

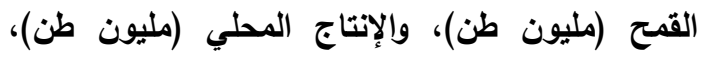
والاستهلاك (مليون طن) من النشرات المختلفة التي لإني

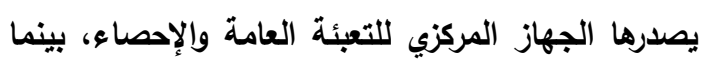

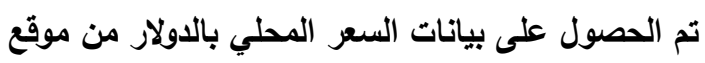

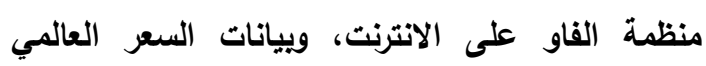

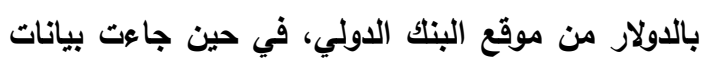
السكان من موقع الأمم المتحدة.

$$
\text { نتائج الاراسة }
$$
1- الأهمية النسبية لقيمة واردات الغذاء من قيمة

$$
\text { الواردات الكلية. }
$$

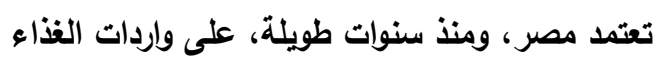
لسد الفجوة الغذائية المتزايدة. لذا، ساهمت الواردات الغذائية، ولعقود عديدة، بنسبة كبيرة في إجمالي الواردات

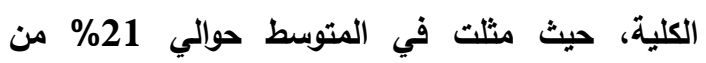
متوسط إجمالي قيمة الواردات الكلية المصرية خلال الفترة (1991-2019)، وذلك كما يتضح من جدول

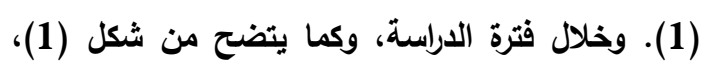
تأرجحت قيمة واردات الغذاء في مصر بين حد أدني بلغ 1 بلغ

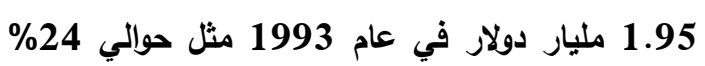
من إجمالي قيمة الواردات في نفس السنة، وحد أعلي بلغ 15.67 مليار دولار في عام 2012 مثل حوالي في 23 من إجمالي قيمة الواردات في نفس السنة. كذلك، ولكار بلغ متوسط قيمة واردات مصر من الغذاء حوالي 7.10 مليار دولار خلال نفس الفترة. إن التكاليف المالية والاقتصادية لسد هذه الفجوة، خاصة بعد تحرير سعر الصرف، تمثل عبئا كبيرا على

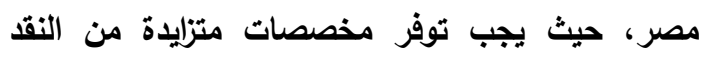

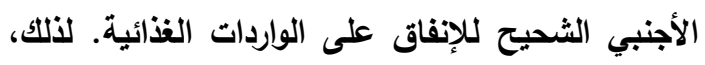


كد أدنى في عام 2000، و14.89 مليون طن كد أعلى في عام 2018، وذلك خلال فترة الدراسة.

\begin{tabular}{|c|c|c|c|c|c|}
\hline \multicolumn{2}{|c|}{ واردات القمح } & \multicolumn{2}{|c|}{ الواردات الغذائية } & \multirow{2}{*}{ 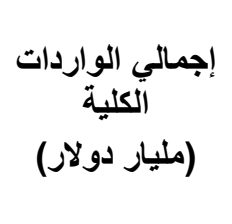 } & \multirow[b]{2}{*}{ السنة } \\
\hline \% الواردات إلغذائية & (مليار دولار) & \% الواردات إجمالي & (مليار دولار) & & \\
\hline 31.02 & 0.62 & 25.43 & 2.00 & 7.86 & 1991 \\
\hline 29.12 & 0.71 & 29.57 & 2.44 & 8.25 & 1992 \\
\hline 25.59 & 0.50 & 23.79 & 1.95 & 8.21 & 1993 \\
\hline 28.95 & 0.76 & 25.90 & 2.64 & 10.19 & 1994 \\
\hline 26.28 & 0.88 & 28.38 & 3.33 & 11.74 & 1995 \\
\hline 32.10 & 1.23 & 29.48 & 3.84 & 13.02 & 1996 \\
\hline 23.73 & 0.82 & 26.19 & 3.46 & 13.21 & 1997 \\
\hline 23.35 & 0.82 & 21.62 & 3.50 & 16.17 & 1998 \\
\hline 16.60 & 0.61 & 22.78 & 3.65 & 16.02 & 1999 \\
\hline 20.27 & 0.71 & 24.14 & 3.52 & 14.58 & 2000 \\
\hline 20.11 & 0.67 & 24.78 & 3.32 & 13.38 & 2001 \\
\hline 23.44 & 0.82 & 27.24 & 3.48 & 12.77 & 2002 \\
\hline 22.26 & 0.61 & 21.04 & 2.73 & 12.95 & 2003 \\
\hline 25.13 & 0.73 & 18.15 & 2.90 & 15.95 & 2004 \\
\hline 23.23 & 0.92 & 17.72 & 3.98 & 22.45 & 2005 \\
\hline 34.85 & 1.37 & 14.38 & 3.93 & 27.30 & 2006 \\
\hline 39.13 & 2.16 & 14.88 & 5.52 & 37.10 & 2007 \\
\hline 27.61 & 2.46 & 18.43 & 8.92 & 48.38 & 2008 \\
\hline 28.48 & 2.20 & 17.19 & 7.73 & 44.95 & 2009 \\
\hline 25.61 & 2.60 & 19.17 & 10.15 & 52.92 & 2010 \\
\hline 21.63 & 3.20 & 25.12 & 14.79 & 58.90 & 2011 \\
\hline 23.55 & 3.69 & 22.64 & 15.67 & 69.20 & 2012 \\
\hline 23.26 & 2.72 & 17.64 & 11.67 & 66.18 & 2013 \\
\hline 21.19 & 3.16 & 22.31 & 14.90 & 66.79 & 2014 \\
\hline 16.78 & 2.42 & 22.64 & 14.39 & 63.57 & 2015 \\
\hline 19.33 & 2.12 & 19.62 & 10.95 & 55.79 & 2016 \\
\hline 19.46 & 2.62 & 21.88 & 13.49 & 61.63 & 2017 \\
\hline 20.54 & 2.64 & 17.82 & 12.83 & 72.00 & 2018 \\
\hline 21.11 & 3.02 & 20.20 & 14.33 & 70.92 & 2019 \\
\hline 23.19 & 1.65 & 20.76 & 7.10 & 34.22 & المتوسط \\
\hline
\end{tabular}


المصدر: جمعت وحسبت من 1- موقع منظمة التجارة العالمية 2- موقع منظمة الفاو موفع منظه لجارة
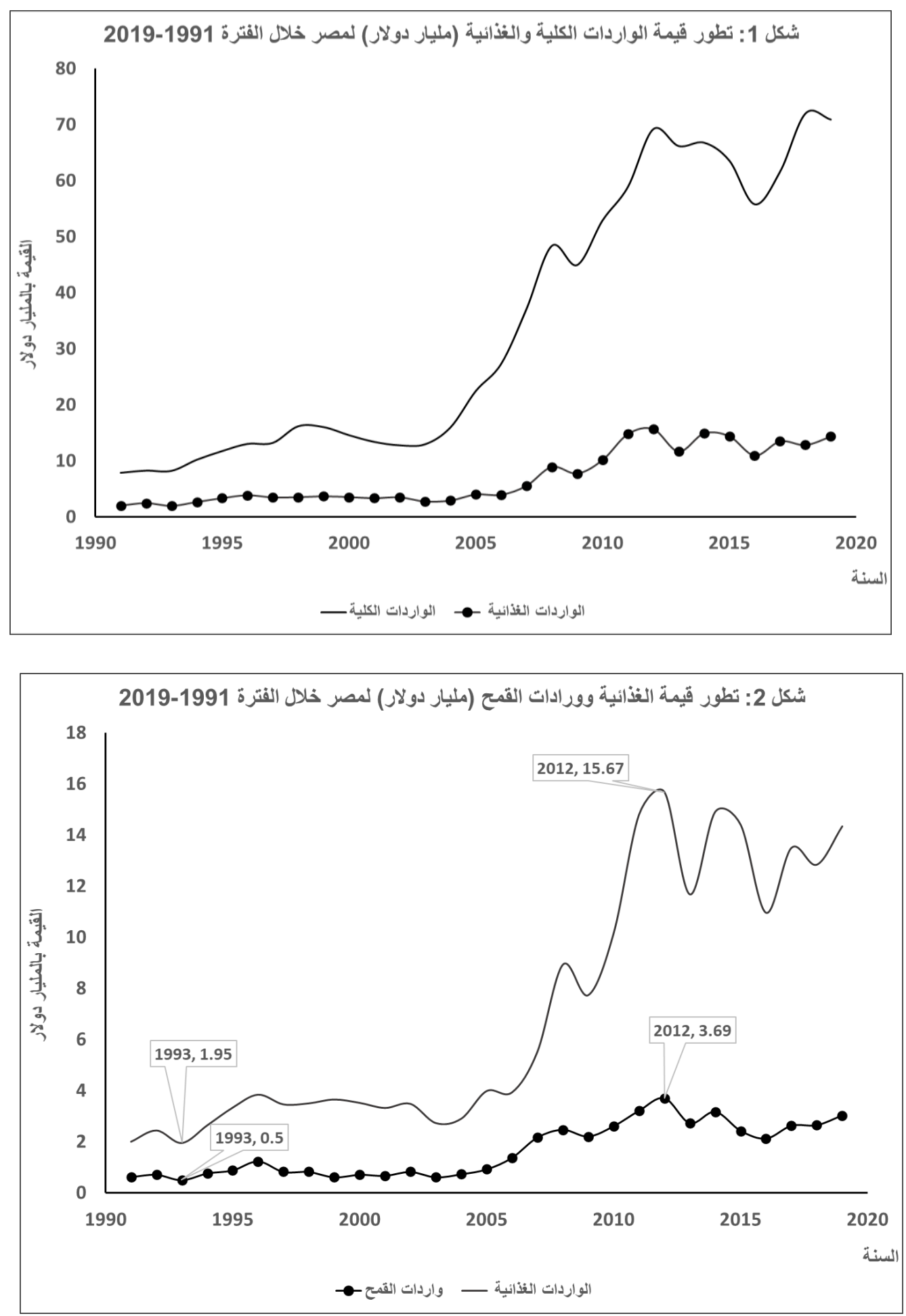

المصدر : بيانات جدول (1) بالدراسة. 
جدول (3). تطور قيمة إجمالي الواردات الغذائية وواردات القمح (مليار دولار) لمصر خلال الفترة (1991-2019).

\begin{tabular}{|c|c|c|c|c|c|c|c|c|}
\hline (مليون نسمة) & السعرية & 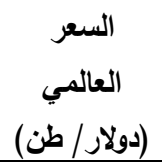 & 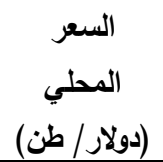 & الاكتفاء & 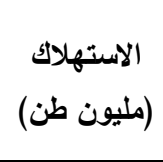 & (الإنتاج & واردات القمح طن & السنة \\
\hline 58.75 & 1.23 & 128.67 & 158.70 & 46.84 & 9.57 & 4.48 & 5.04 & 1991 \\
\hline 60.04 & 1.05 & 151.20 & 158.70 & 49.50 & 9.33 & 4.62 & 4.94 & 1992 \\
\hline 61.28 & 1.13 & 140.24 & 157.80 & 51.54 & 9.38 & 4.83 & 4.88 & 1993 \\
\hline 62.50 & 1.05 & 149.73 & 157.50 & 48.90 & 9.07 & 4.44 & 3.95 & 1994 \\
\hline 63.71 & 0.93 & 176.98 & 165.10 & 51.35 & 11.14 & 5.72 & 5.81 & 1995 \\
\hline 64.93 & 0.91 & 207.60 & 188.70 & 48.16 & 11.91 & 5.74 & 4.96 & 1996 \\
\hline 66.15 & 1.23 & 159.48 & 195.90 & 54.54 & 10.72 & 5.85 & 4.85 & 1997 \\
\hline 67.38 & 1.59 & 126.13 & 200.70 & 53.51 & 11.39 & 6.09 & 5.77 & 1998 \\
\hline 68.63 & 1.81 & 112.04 & 202.90 & 56.00 & 11.33 & 6.35 & 4.59 & 1999 \\
\hline 69.91 & 1.75 & 114.09 & 199.60 & 61.69 & 10.64 & 6.56 & 3.85 & 2000 \\
\hline 71.23 & 1.39 & 126.81 & 176.40 & 59.52 & 10.51 & 6.25 & 4.41 & 2001 \\
\hline 72.59 & 1.08 & 148.08 & 159.60 & 53.33 & 12.42 & 6.62 & 5.58 & 2002 \\
\hline 73.98 & 0.89 & 146.14 & 129.90 & 62.46 & 10.96 & 6.84 & 4.06 & 2003 \\
\hline 75.38 & 1.03 & 156.88 & 161.40 & 61.10 & 11.75 & 7.18 & 4.27 & 2004 \\
\hline 76.78 & 1.27 & 152.35 & 193.80 & 61.16 & 13.31 & 8.14 & 5.63 & 2005 \\
\hline 78.16 & 1.02 & 192.04 & 196.60 & 56.41 & 14.67 & 8.27 & 5.82 & 2006 \\
\hline 79.54 & 0.80 & 255.21 & 204.60 & 53.51 & 13.79 & 7.38 & 5.92 & 2007 \\
\hline 80.95 & 1.44 & 326.03 & 469.90 & 54.84 & 14.55 & 7.98 & 4.08 & 2008 \\
\hline 82.47 & 1.30 & 224.07 & 290.90 & 74.44 & 11.45 & 8.52 & 4.06 & 2009 \\
\hline 84.11 & 1.44 & 223.58 & 322.80 & 40.58 & 17.69 & 7.18 & 9.71 & 2010 \\
\hline 85.90 & 1.25 & 316.26 & 395.30 & 49.01 & 17.15 & 8.41 & 9.8 & 2011 \\
\hline 87.81 & 1.33 & 313.24 & 416.40 & 55.73 & 15.78 & 8.80 & 6.54 & 2012 \\
\hline 89.81 & 1.21 & 312.25 & 376.50 & 56.72 & 16.68 & 9.46 & 6.79 & 2013 \\
\hline 91.81 & 1.36 & 284.89 & 387.60 & 49.37 & 17.83 & 8.80 & 8.74 & 2014 \\
\hline 93.78 & 1.75 & 204.45 & 358.80 & 49.11 & 19.56 & 9.61 & 10.54 & 2015 \\
\hline 95.77 & 1.66 & 166.63 & 277.20 & 47.69 & 19.59 & 9.34 & 12.66 & 2016 \\
\hline 97.75 & 1.22 & 174.20 & 212.00 & 34.05 & 24.73 & 8.42 & 12.03 & 2017 \\
\hline 99.71 & 1.01 & 209.93 & 212.20 & 35.45 & 23.55 & 8.35 & 14.89 & 2018 \\
\hline 101.67 & 1.33 & 201.69 & 268.33 & 40.30 & 21.24 & 8.56 & 13.02 & 2019 \\
\hline 78.02 & 1.25 & 193.13 & 241.24 & 52.3 & 14.20 & 7.20 & 6.8 & المتوسط \\
\hline
\end{tabular}


https://www.un.org/development/desa/pd 4

5ttps://www.worldbank.org/en/research/commodity-markets موقع البنك الدولي

كذلك فقد تم الكثف عن أخطاء القياس بالنموذج مثل الارتباط الاتي Serial correlation، ومشكلة عدم ثبات تباين حد الخطأ Heteroskedasticity، Normality of ومشكلة تبعية البواقي للتوزيع الطبيعي Residuals، وثبت خلو النموذج من تلك المشكلات.

$$
\text { أولا: نسبة الاكتفاء الذاتي }
$$

تعتبر نسبة الاكتفاء الأتي من أهم العوامل المؤثرة على كمية واردات مصر من القمح خلال القترة (19912019)، حيث إن زيادة نسبة الاكتفاء الذاتي بنسبة ماندان 1\%، تؤدي إلى انخفاض كمية واردات مصر من القمح بنسبة 1.086\%، مع ثبات العوامل الأخرى على ما هي عليه، وذلك بحسب بيانات جدول (4). لألك، ركزت الاستراتيجيات والسياسات الزراعية الحالية على رفع نسب الاكتفاء الذاتي من المحاصيل الاستراتيجية التي تشكل وارداتها نسبة كبيرة من واردات مصر الغذائية، مثل من الاهن القمح والأرة، حيث هدفت "استراتيجية التنمية الزراعية المستدامة نحو عام 2030" إلى رفع نسبة الاكتفاء الأتي من القمح إلى 74\% في عام 2017، 81\% بحلول عام 2030 (MALR, 2009 (إل

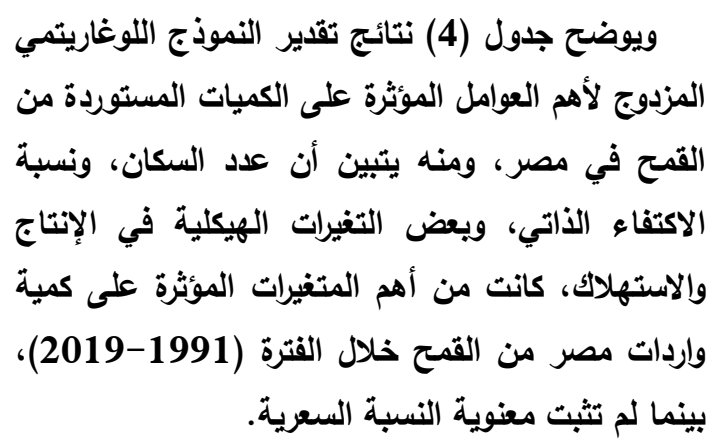
statistic للتقدير S.E. of regression منخفضة ويلغت حوالي 0.142، مما يدل علي دقة النموذج المستخدم. كذلك فإن قيمة معامل التحديد المعدل بلغت حوالي 0.88، مما يعني أن 88\% من التغيرات في كمية واردات مصر من القمح خلال الفترة (1991-2019) ترجع إلى المتغيرات التي تضمنها النموذج، والباقي يرجع لعوامل أخري غير مقاسة. جدول (4). نتائج تقدير النموذج اللوغاريتمي المزدوج: كمية واردات القمح (1991-2019).

\begin{tabular}{lcccc}
\hline \multirow{2}{*}{ Variable } & \multicolumn{4}{c}{ Wheat imports (Million tons) } \\
\cline { 2 - 5 } & Coefficient & Std. Error & t-Statistic & p-value \\
\hline Intercept & 2.317 & 1.326 & 1.748 & 0.093 \\
LnPop $_{i}$ & 0.851 & 0.307 & 2.771 & 0.0106 \\
LnSr $_{i}$ & -1.086 & 0.217 & -4.995 & 0.0000 \\
LnPr $_{i}$ & 0.019 & 0.138 & 0.137 & 0.893 \\
$D$ & 0.282 & 0.127 & 2.226 & 0.0356 \\
\hline $\mathbf{R}^{2}$ & 0.897 & Akaike info criterion & -.909 & \\
Adjusted R & 0.88 & Schwarz criterion & -.673 & \\
F-statistic & 52.312 & Hannan-Quinn criter. & -.835 &
\end{tabular}




\begin{tabular}{lccc}
\hline$p$-value (F-statistic) & 0.00000 & Durbin-Watson stat & 2.417 \\
S.E. of regression & 0.142 & & \\
\hline
\end{tabular}

مع ثبات العوامل الأخرى على ما هي عليه، فإن زيادة عدد السكان بنسبة 1\% تؤدي إلي زيادة كمية واردات مصر من القمح بنسبة 0.851\%. وتوضح إحصائيات السكان أن عدد سكان مصر يزداد بشكل كبير، فقد ارتفع معدل النمو السكاني من 2.04٪ سنويأيات خلال الفترة 1996-2006 إلى 56 2.56٪ سنويا خلال القترة 2006-2017 (الجهاز المركزي للتعبئة العامة والإحصاء، 2017). وتوضح بيانات جدول (3) أن عدد سكان مصر بلغ حوالي 58.75 مليون نسمة في عام

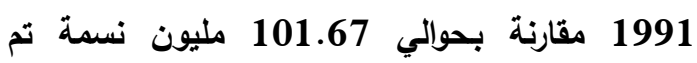
إحصاؤهم في عام 2019، ويزيادة بلغت حوالي 73 عن عدد السكان في عام 1991. علاوة على ذلك، توقعت الأمم المتحدة أن يرتفع عدد سكان مصر إلى 123 مليونًا بحلول عام 2030، ثم إلى 174 مليونًا بحلول عام 2050، بافتراض ثبات معدلات الخصوية والوفيات على ما هي عليه (الأمم المتحدة، 2017). إن التفاوت الكبيز بين الإنتاج المحلي والاستهلاك من القمح خلال فترة الدراسة يرجع إلى الزيادة الكبيرة والسريعة في الطلب على القمح بسبب زيادة معدلات النمو السكاني، بالإضافة إلى أنماط الاستهلاك المنتشرة،

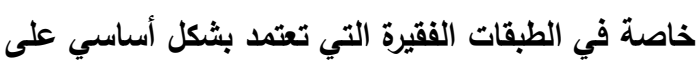
الأغذية منخفضة التكلفة والغتية بالسعرات الحرارية مثل الخبز (Bordignon, 2013). في نفس الوقت، أدت محدودية وندرة الموارد الزراعية من الأراضي الزراعية الخصبة والموارد المائية إلى عجز الإنتاج المحلي عن تلبية الزيادة في الطلب على القمح. كما سيؤدي النمو السكاني السريع إلى تفاقم مشكلة انعدام الأمن الغذائي ليس فقط عن طريق زيادة الطلب على الغذاء، ولكن أيضًا من خلال زيادة الضغط على الموارد الزراعية لإنتاج المزيد من الغذاء، بالإضافة إلى زيادة الزحف العمراني علي الأراضي الزراعية.
وعلي الرغم من أن نسبة الاكتفاء الأتي من القمح بلغت 74.44\% كد أقصي في عام 2009، إلا أنها تراجعت بلرجة كبيرة بلعا من عام 2010، حيث إنه خلال القترة (2010-2019) لم تتجاوز نسبة الاكتفاء الأتي 50\% إلا في عامي 2012، 2013، ثم تناقصت بعد ذلك حتى وصلت إلي أدني قيمة لها ويلغت حوالي 34.05 في عام 2017، وذلتك كما توضحه بيانات جدول (3). ويصفة عامة، حققت نسبة الاكتفاء الأتي من القمح متوسطا سنويا بلغ حوالي 50.72\% خلال

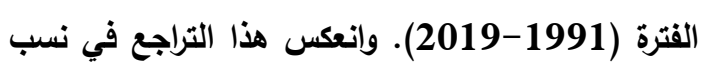
الاكتفاء الذاتي على الكميات المستوردة من القمح، حيث تضاعف الكميات التي تستوردها مصر خلال العشر سنوات الأخيرة (2010-2019) مقارنة بالسنوات السابقة، حتى وصلت إلي أقصي قيمة لها ويلغت حوالي 14.89 مليون طن في عام 2018.

ويعزي هذا التناقص الكبير في نسب الاكتفاء الأتي من القمح خلال القترة (2010-2019) إلى عجز فئ فئ الإنتاج المحلي من القمح عن الزيادة بنفس السرعة التي

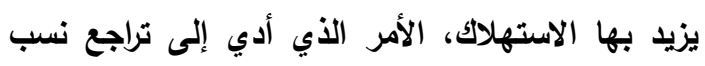
الاكتفاء الأتي، ويالتالي زيادة الفجوة الغذائية من عام الأئ

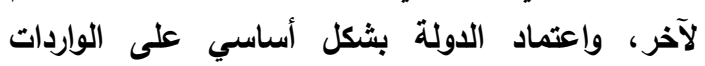
الغذائية لسد تلك الفجوة. ويدراسة بيانات جدول (3)، يتبين تزايد الكميات المستهلكة من القمح عاما بعد الآخر حتى وصلت لأقصي قيمة لها في عام 2017 بلغت 24.73 مليون طن، تمثل حوالي 273\% من الحد الأدنى للكمية المستهلكة من القمت خلائ لمن الفترة

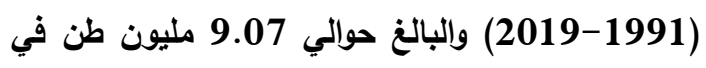
عام 1994، في حين بلغت أقصى كمية منتجة من فن في في في

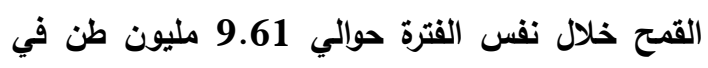

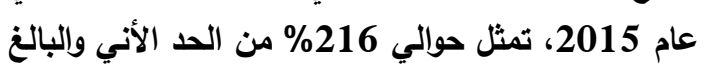
حوالي 4.44 مليون طن في عام 1994. ثانيا: عدد السكان 
المحلية والعالمية للقمح. وهذا يعني أنه مع زيادة عدد

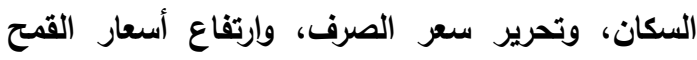

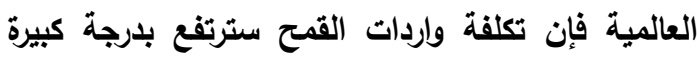
McGill et al, 2015)

$$
\text { المراجع }
$$

1- الجهاز المركزي للتعيئة العامة والإحصاء، نشرات مختلفة، أعداد متفرقة.

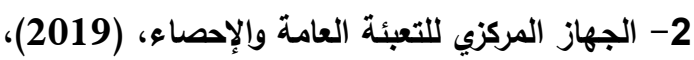
أهم مؤثرات بحث الاخثل والإنفاق والاستهلاك

2017/ 2018، إصدار يونيو 2019، القاهرة.

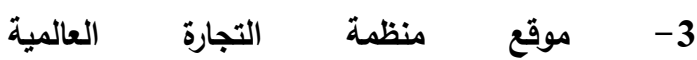
. /https://www.wto.org

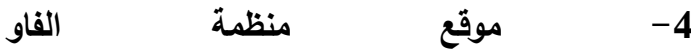

.http://www.fao.org/faostat/en/\#home

$$
\text { 5- موقع البنك الدولي }
$$

https://www.worldbank.org/en/researc

h/commodity-markets

6- 6نظمة الأمم المتحدة،

https://www.un.org/development/desa

/pd

7- Bordignon, G. (2013). The status of poverty and food security in Egypt: analysis and policy recommendations. Cairo: World Food Programme.

8- McGill, J., Prikhodko, D., Sterk, B., \& Talks, P. (2015). Egypt: Wheat sector review. FAO Investment Centre. Country Highlights (FAO) eng no. 21.

9- Metz, H. C., \& Egypt, H. (1990). A Country Study. In GPO for the Library of Congress: Washington, DC, USA.

10- Ministry of Agriculture and Land Reclamation (MALR). (2009). Sustainable Agricultural Development Strategy Towards 2030, Cairo, Egypt.
ثالثا: التغيرات الهيكلية

لقياس أثر التغيرات الهيكلية، تم استخدام متغير وهمي Dummy Variable يأخذ القيمة "صفر" للسنوات بدءا من 1991 حتى 2009، والقيمة "واحد" للسنوات بدءا من 2010 حتى 2019. ويعتبر تغير أنماط الاستهلاك في الفترة الثانية (2010-2019) مقارنة بالفترة الأولي (1991-2009) من أهم التغيرات الهيكلية، نتيجة لزيادة نسبة الفقر التي قفزت من

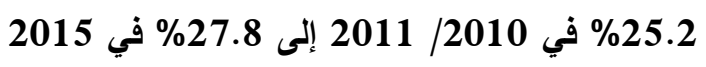
ثم إلى 32.5\% في 2017/ 2018 \% مقارنة بحوالي

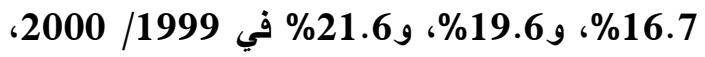
و2004/ 2005، و2008/ 2009 على الترتيب (الجهاز المركزي للتعبئة العامة والإحصاء، 20192005)، الأمر الذي أدي إلي زيادة الكميات المستهلكة من الخبز

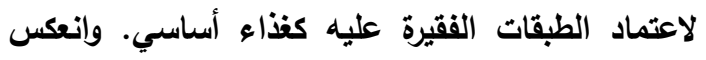
هذا على متوسط استهلاك القرد السنوي من القمح الذي

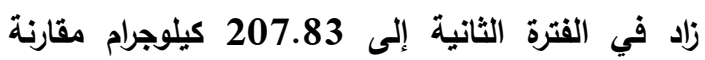
بحوالي 165.46 كيلوجرام في الفترة الأولى ويزيادة قدرها 25.61\%. هذا بالإضافة إلى التغيرات السياسية بعد عام 2010 وما صاحبها من تغيرات اقتصادية كبيرة. وتثير بيانات جدول (4) إلى أن التغيرات الهيكلية

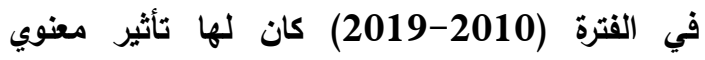
إحصائيا علي زيادة كمية الواردات السنوية من القمح خلال تلكت الفترة والتي بلغت في المتوسط حوالي 10.47 مليون طن مقارنة بحوالي 4.87 مليون طن متوسط كمية واردات القمح خلال الفترة الأولي (1991.2009

$$
\text { رابعا: النسبة السعرية }
$$

لم تثبت معنوية النسبة السعرية، وريما يرجع ذلك الك الكيه إلى أن الهذف الأساسي لمستورد القمح المصري هو سد الفجوة الغذائية من القمح، ومن ثم استيراد الكمية التي التي تكفي لسد احتياجات السكان بعض النظر عن الأسعار 


\title{
AN ANALYTICAL STUDY FOR MAIN FACTORS AFFECTING WHEAT IMPORTS TO EGYPT
}

\author{
H. S. A. Abdelaal and A. M. El-Shafei \\ Agricultural Economics Department, Faculty of Agriculture, Minia University
}

\begin{abstract}
Wheat, the most significant agricultural crop in Egypt, is one of the leading food imports. For decades, Egypt relied heavily on wheat imports to bridge the food gap resulting from the shortfall in domestic production to meet the increasing consumption of wheat. Thus, the study primarily aimed to identify and study the key factors affecting the quantities of wheat imports. The study utilized the multiple linear regression model, estimating the double-log model. The results showed that wheat imports represented a large share of food imports, amounting to an average of $23.19 \%$ during (1991-2019). The regression estimation results suggested that the self-sufficiency ratio of wheat and the population were the major determinants of Egyptian wheat imports quantities during the study period. Holding other factors constant, as the self-sufficiency rate of wheat increases by $1 \%$, wheat imports quantity decreases by $1.08 \%$, and as the population increases by $1 \%$, wheat imports quantity increases by $0.86 \%$. Therefore, the study recommends rationalizing wheat consumption and working to raise the domestic production of wheat.
\end{abstract}

Key words: Wheat imports - self-sufficiency ratio - Population- Food gap 
أسماء السادة المحكمين

أ.د/ ياسر عبدالحميد عبدالمرضى دياب لية الزراعة - جامعة أسيوط

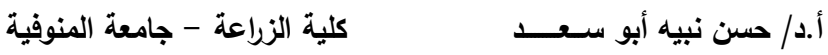

